\title{
A DISCRETE METHOD FOR THE LOGARITHMIC-KERNEL INTEGRAL EQUATION ON AN OPEN ARC
}

\author{
S. PRÖSSDORF ${ }^{1}$, J. SARANEN ${ }^{2}$ and I. H. SLOAN ${ }^{3}$
}

(Received 12 June 1991; revised 10 December 1991)

\begin{abstract}
Here we discuss the stability and convergence of a quadrature method for Symm's integral equation on an open smooth arc. The method is an adaptation of an approach considered by Sloan and Burn for closed curves. Before applying the quadrature scheme, we use a cosine substitution to remove the endpoint singularity of the solution. The family of methods includes schemes with any order $O\left(h^{p}\right)$ of convergence.
\end{abstract}

\section{Introduction}

Let $\Gamma$ be a smooth open arc in the plane. We consider the numerical solution of the integral equation

$$
-\frac{1}{\pi} \int_{\Gamma} v(y) \log |x-y| d s_{y}=g(x), \quad x \in \Gamma .
$$

Equation (1.1) arises for example in electrostatics, fluid dynamics and in simulation of cracks. Computationally efficient quadrature methods for the solution of (1.1) have recently been introduced and analysed in the case of a closed curve. In [13] Sloan and Burn introduced a family of "unconventional" quadrature methods. This approach was further developed and analysed by Saranen and Sloan [10]. A different quadrature method for a closed curve, involving subtraction of the singularity, was analysed by Saranen in [9]. On the other hand, quadrature methods for Cauchy-singular integral equations on open curves were investigated by Rathsfeld [8], by Prössdorf and Rathsfeld

\footnotetext{
${ }^{1}$ Karl-Weierstrass-Institut für Mathematik, Mohrenstrasse 39, D-O 1086 Berlin, Germany.

${ }^{2}$ Section of Mathematics, Faculty of Technology, University of Oulu, 90570 Oulu, Finland.

${ }^{3}$ School of Mathematics, University of New South Wales, Sydney, N.S.W. 2033, Australia.

(C) Copyright Australian Mathematical Society 1993, Serial-fee code 0334-2700/93
} 
[5], and by Prössdorf and Sloan [7]. For some applications of (1.1), see e.g. [2].

Here we propose a quadrature method which is an adaptation of the quadrature method introduced by Sloan and Burn [13] for the closed-curve case. To be more precise, the quadrature method is here applied after the well-known cosine substitution. The cosine substitution was introduced already by Multhopp (1938) in [4] for the airfoil equation of Prandtl, see also [6, Section 3.4] and [11],[12],[16]. For Symm's equation (1.1) it was recently used by Yan and Sloan in [17]. It is well known that even for a smooth righthand side $g$ the solution $v$ of (1.1) may have the singularity $O\left(|x-c|^{-1 / 2}\right)$ at the endpoint $c$ of the arc $\Gamma$. The cosine substitution removes this bad behaviour of the solution and therefore enables us to construct numerical schemes with good stability and convergence properties. As in the case of a smooth closed curve (see [10],[13]), the family of quadrature methods in this paper includes schemes of arbitrarily high, but fixed order $O\left(h^{p}\right)$ of convergence. In particular, we describe explicitly a simple method (see Example 4.1) which is stable and has the order $O\left(h^{3}\right)$ of convergence; the discretisation parameter $h$ is inversely proportional to the number of the meshpoints on $\Gamma$.

The cosine substitution has already been used for numerical solution of (1.1) by Atkinson and Sloan in [1], by Joe and Yan in [3], and by Sloan and Stephan in [15]. Atkinson and Sloan discuss a completely discrete method which is based on a further discretisation of the Galerkin method with trigonometric trial functions. Joe and Yan, and Sloan and Stephan, analyse collocation methods, but only in the case of an open interval instead of a general arc. Other methods were described by Costabel et al. in [2] and by Sloan and Spence in [14]. All in all, from the above articles only the work [1] of Atkinson and Sloan (in addition to our paper) gives a fully discrete system of equations for a general open arc. Therefore we compare our result to that of [1] in some detail in Section 4.

\section{Preliminaries}

For the convenience of the reader, we use the notations of Yan and Sloan [17]. We assume that the arc $\Gamma$ has the parametrisation

$$
\nu(\xi)=\left(\nu_{1}(\xi), \nu_{2}(\xi)\right), \quad-1 \leq \xi \leq 1,
$$

such that $\xi \mapsto \nu(\xi)$ is infinitely differentiable with $\left|\nu^{\prime}(\xi)\right|>0,-1 \leq \xi \leq$ 1. As the only restriction on generality, we impose the condition that the capacity of the arc $\Gamma$ differs from one. By this assumption (1.1) is uniquely 
solvable. For a detailed discussion of the capacity we refer to Yan and Sloan [17]. We apply the cosine transformation given by $\xi=\cos t, 0 \leq t \leq \pi$, and define $a:[0, \pi] \rightarrow \Gamma$ by $a(t)=\nu(\cos t)$. Now (1.1) becomes

$$
-\frac{1}{\pi} \int_{0}^{\pi} u(\tau) \log |a(t)-a(\tau)| d \tau=f(t), \quad 0 \leq t \leq \pi,
$$

where

$$
\left\{\begin{array}{l}
u(t)=v(a(t))\left|\nu^{\prime}(\cos t) \| \sin t\right| \\
f(t)=g(a(t))
\end{array}\right.
$$

By the formulae above, the functions $a(t), u(t)$ and $f(t)$ may be considered as $2 \pi$-periodic even functions defined on the whole real line $\mathbb{R}$. For the following, it is convenient to consider (2.2) as an integral equation for $2 \pi$ periodic even functions $u$ and $f$ in the form

$$
\left(L_{e} u\right)(t)=f(t), \quad-\pi \leq t \leq \pi,
$$

where

$$
\left(L_{e} u\right)(t)=-\frac{1}{2 \pi} \int_{-\pi}^{\pi} u(\tau) \log |a(t)-a(\tau)| d \tau, \quad-\pi \leq t \leq \pi .
$$

For the equivalence of (2.2) and (2.4), it is sufficient to observe that the kernel function

$$
-\frac{1}{\pi} \log |a(t)-a(\tau)|
$$

is also $2 \pi$-periodic and even with respect to both the arguments $t$ and $\tau$.

In our analysis of the numerical method, we shall make use of the decomposition

$$
L_{e} u=A_{e} u+B_{e} u,
$$

with

$$
\left(A_{e} u\right)(t)=-\frac{1}{2 \pi} \int_{-\pi}^{\pi} u(\tau) \log \left[2 e^{-1}|\cos t-\cos \tau|\right] d \tau
$$

and

$$
\left(B_{e} u\right)(t)=\int_{-\pi}^{\pi} u(\tau) b_{e}(t, \tau) d \tau
$$

where

$$
b_{e}(t, \tau)=\left\{\begin{array}{l}
-\frac{1}{2 \pi} \log \left|\frac{e}{2} \cdot \frac{a(t)-a(\tau)}{\cos t-\cos \tau}\right|, \quad t \neq-\tau, \tau(\bmod 2 \pi), \\
-\frac{1}{2 \pi} \log \left|\frac{e}{2} \nu^{\prime}(\cos t)\right|, \quad t=-\tau, \tau(\bmod 2 \pi) .
\end{array}\right.
$$

We notice that $b_{e}(t, \tau)$ is a smooth function of $t$ and $\tau$ on $\mathbb{R} \times \mathbb{R}$ and is $2 \pi$-periodic and even with respect to each variable, i.e. $b_{e} \in C_{2 \pi, e}^{\infty}(\mathbb{R} \times \mathbb{R})$. 
LEMMA 2.1. If $u$ is even then

$$
\left(A_{e} u\right)(t)=-\frac{1}{\pi} \int_{-\pi}^{\pi} u(\tau) \log \left|2 e^{-\frac{1}{2}} \sin \left(\frac{t-\tau}{2}\right)\right| d \tau .
$$

Proof. Since

it follows from (2.7) that

$$
\cos t-\cos \tau=2 \sin \frac{t+\tau}{2} \sin \frac{\tau-t}{2}
$$

$$
\begin{aligned}
\left(A_{e} u\right)(t)= & -\frac{1}{2 \pi} \int_{-\pi}^{\pi} u(\tau) \log \left[4 e^{-1}\left|\sin \frac{t+\tau}{2} \sin \frac{t-\tau}{2}\right|\right] d \tau \\
= & -\frac{1}{2 \pi} \int_{-\pi}^{\pi} u(\tau) \log \left|2 e^{-1 / 2} \sin \frac{t+\tau}{2}\right| d \tau \\
& -\frac{1}{2 \pi} \int_{-\pi}^{\pi} u(\tau) \log \left|2 e^{-1 / 2} \sin \frac{t-\tau}{2}\right| d \tau .
\end{aligned}
$$

On making the substitution $\tau=-\tau^{\prime}$ in the first integral and then using the fact that $u$ is even, the result follows immediately.

The operator $A_{e}$ admits a canonical continuation $A$ to the whole space of $L^{2}$-functions on $[-\pi, \pi]$, defined by

$$
(A u)(t)=-\frac{1}{\pi} \int_{-\pi}^{\pi} u(\tau) \log \left|2 e^{-\frac{1}{2}} \sin \left(\frac{t-\tau}{2}\right)\right| d \tau .
$$

The operator $A$ can be identified with Symm's integral operator on the circle with the radius $\rho=e^{-1 / 2}$. The aforementioned fact that the operators $A_{e}$ and $A$ coincide in the space of the $2 \pi$-periodic even functions provides the crucial point of our analysis (as indeed it does for most or all of the methods which employ the cosine transformation). More precisely, in the case where the arc $\Gamma$ is the interval $\left[-2 e^{-1}, 2 e^{-1}\right]$, our method turns out to be equivalent to the method of Sloan and Burn [13] for the circle with the radius $\rho=e^{-1 / 2}$.

In the following, the general case of the operator $L_{e}$ will be reduced to the case $L_{e}=A_{e}$ by using a perturbation argument.

The equation (2.4) will be considered in Sobolev spaces of $2 \pi$-periodic even functions. These spaces $H_{e}^{s}, s \in \mathbb{R}$, consist of $2 \pi$-periodic even functions $u$ (more precisely, distributions)

$$
u(t)=\frac{1}{\sqrt{2 \pi}} \sum_{m=-\infty}^{\infty} \hat{u}(m) e^{i m t}
$$

satisfying

$$
\|u\|_{s}:=\left(|\hat{u}(0)|^{2}+\sum_{m \neq 0}|m|^{2 s}|\hat{u}(m)|^{2}\right)^{1 / 2}<\infty,
$$


where

$$
\hat{u}(m)=\frac{1}{\sqrt{2 \pi}} \int_{-\pi}^{\pi} u(t) e^{-i m t} d t .
$$

For $u \in H_{e}^{s}$ there is also the cosine type representation

$$
u(t)=\sqrt{\frac{2}{\pi}}\left(\sum_{m=1}^{\infty} \hat{u}^{c}(m) \cos m t+\frac{1}{2} \hat{u}^{c}(0)\right)
$$

with the Fourier cosine coefficients $\hat{u}^{c}(m)$ defined by

$$
\hat{u}^{c}(m)=\sqrt{\frac{2}{\pi}} \int_{0}^{\pi} u(t) \cos m t d t .
$$

It is easily seen that $\hat{u}(m)=\hat{u}^{c}(m)$ for $u \in H_{e}^{s}$ because $u$ is even. The norm in $H_{e}^{s}$ can therefore be expressed alternatively as

$$
\|u\|_{s}=\left(\left|\hat{u}^{c}(0)\right|^{2}+\sum_{m=1}^{\infty}|m|^{2 s}\left|\hat{u}^{c}(m)\right|^{2}\right)^{1 / 2} \text {. }
$$

The usual Sobolev spaces $H^{S}$ of $2 \pi$-periodic functions on $\mathbb{R}$ and the corresponding norms are defined by the relations (2.12)-(2.14) without the restriction that the distribution $u$ is even. By our assumption on $\Gamma$, the operator $L_{e}$ defines an isomorphism $L_{e}: H_{e}^{s} \rightarrow H_{e}^{s+1}$ for all $s \in \mathbb{R}$, see Yan and Sloan [17].

\section{Description of the method}

Our numerical method for the approximate solution of the equation

$$
\left(L_{e} u\right)(t)=f(t), \quad-\pi \leq t \leq \pi
$$

(with $f$ and $u$ even functions) is closely related to the method proposed by Sloan and Burn in [13]. The method yields a fully discretised system of equations, where the unknowns are approximations of the solution $u(t)$ at the nodal points of a uniform mesh. Our method consists of a suitable discretisation of a certain Petrov-Galerkin approximation.

First we define a partition of the interval $I=[-\pi, \pi]$. For this let $N=$ $2 L+1, L \geq 1$, so that $N$ is an odd integer. We define the uniform mesh $\left\{t_{j}\right\}_{j=0}^{N}$ by

$$
t_{j}=-\pi+j h, \quad 0 \leq j \leq N,
$$

with the mesh parameter $h=2 \pi / N$. 
Next we introduce the trapezoidal approximation $L_{e, h}$ of the operator $L_{e}$, defined by

$$
\left(L_{e, h} u\right)(t)=-\frac{h}{2 \pi} \sum_{j=0}^{N} \log \left|a(t)-a\left(t_{j}\right)\right| u\left(t_{j}\right) .
$$

The notation $\Sigma^{\prime \prime}$ in (3.3) means

$$
\sum_{j=0}^{N}{ }^{\prime \prime} c_{j}=\frac{1}{2}\left(c_{0}+c_{N}\right)+\sum_{j=1}^{N-1} c_{j},
$$

a widely used convention. In our Petrov-Galerkin method we use cosine functions as trial functions. First let $T^{h}$ be the finite-dimensional space of all trigonometric polynomials of degree $\leq L$, that is

$$
T^{h}=\left\{v(t)=\sum_{-L}^{L} c_{n} e^{i n t}: c_{n} \in \mathbb{C}\right\} .
$$

The space $T_{e}^{h}$ of cosine functions is defined by

$$
T_{e}^{h}=\left\{v(t)=\sum_{n=0}^{L} a_{n} \cos n t: a_{n} \in \mathbb{C}\right\} .
$$

Note that the space $T_{e}^{h}$ can be characterised as the "even part" of $T^{h}$, i.e. the function $v$ belongs to $T_{e}^{h}$ if and only if $v$ is even and $v \in T^{h}$. This property is one of the crucial points in our approach. Correspondingly, as test functions we use "even parts" of the usual $2 \pi$-periodic spline spaces $S^{d}$. To be more precise, we define

$$
S^{d}=\left\{v: \mathbb{R} \rightarrow \mathbb{C} ; v \text { is } 2 \pi \text {-periodic, }\left.v\right|_{\left(t_{j}, t_{j+1}\right)} \in \mathscr{P}^{d}, v \in C^{d-1}\right\},
$$

where $\mathscr{P}^{d}$ is the space of polynomials of degree $d$, and decompose (direct sum)

$$
S^{d}=S_{e}^{d}+S_{o}^{d},
$$

where

$$
\begin{gathered}
S_{e}^{d}=\left\{v \in S^{d}: v \text { is even }\right\}, \\
S_{o}^{d}=\left\{v \in S^{d}: v \text { is odd }\right\} .
\end{gathered}
$$

It is easy to see that the decomposition (3.7) really exists. Furthermore, the spaces in (3.7) have the dimensions

$$
\operatorname{dim} S^{d}=N, \quad \operatorname{dim} S_{e}^{d}=L+1, \operatorname{dim} S_{o}^{d}=L .
$$



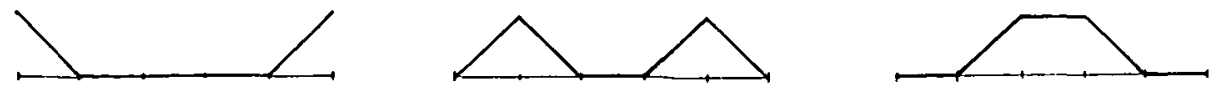

Figure 1
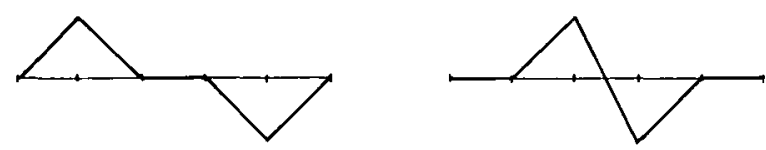

FIGURE 2

For our testspace we choose $S_{e}^{d}$. (Observe that the spaces $T_{e}^{h}$ and $S_{e}^{d}$ have the same dimension.) For the practical implementation of the method we need a concrete set of basis functions in $S_{e}^{d}$. Let us illustrate this point in the case of the piecewise linear continuous functions. Let $v_{j} \in S^{1}$ be the usual hat-functions (Courant's functions) satisfying

$$
v_{j}\left(t_{k}\right)= \begin{cases}1, & k=j \\ 0, & k \neq j\end{cases}
$$

The space $S_{e}^{1}$ has a basis $\left\{v_{j, e}\right\}_{0}^{L}$ given by

$$
\left\{\begin{array}{l}
v_{j, e}=v_{j}+v_{N-j}, \quad 1 \leq j \leq L, \\
v_{0, e}=v_{0}
\end{array}\right.
$$

and the space $S_{o}^{1}$ has a basis $\left\{v_{j, o}\right\}_{1}^{L}$, where

$$
v_{j, o}=v_{j}-v_{N-j}, \quad 1 \leq j \leq L .
$$

Since $\left\{v_{j}\right\}_{0}^{N-1}$ is a basis of $S^{1}$, the decomposition (3.7) is also proved. For $L=2$, the graphs of the functions $v_{j, e}$ and $v_{j, o}$ are shown in the Figures 1 and 2 .

Our method is defined as a full discretisation of the following PetrovGalerkin method: find $u_{h} \in T_{e}^{h}$ with

$$
\left(L_{e} u_{h}, v\right)=(f, v), \quad v \in S_{e}^{d} .
$$

To carry out the discretisation, we replace the operator $L_{e}$ by the operator $L_{e, h}$ and use a discrete inner product instead of the $L^{2}$-inner product

$$
(u, v)=\int_{-\pi}^{\pi} u(t) \overline{v(t)} d t
$$

Some notations are needed. In the interval $\left[-\frac{1}{2}, \frac{1}{2}\right]$, we fix a symmetric quadrature rule

$$
\int_{-\frac{1}{2}}^{\frac{1}{2}} f(t) d t \simeq \sum_{j=1}^{M} w_{j}\left(f\left(\eta_{j}\right)+f\left(-\eta_{j}\right)\right)=: Q(f),
$$


with the points $\eta_{j}$ such that $0 \leq \eta_{1}<\cdots<\eta_{M}<\frac{1}{2}$ and with the weights $w_{j}$ satisfying

$$
\sum_{j=1}^{M} w_{j}=\frac{1}{2}, \quad w_{j}>0, \quad 1 \leq j \leq M .
$$

The discrete inner product $(u, v)_{h}$ is given by

$$
(u, v)_{h}=Q_{h}(u \bar{v}),
$$

where $Q_{h}$ is the composite quadrature rule for the interval $[-\pi, \pi]$,

$$
Q_{h}(f):=h \sum_{k=0}^{N-1} \sum_{j=1}^{M} w_{j}\left[f\left(\left(k+\frac{1}{2}+\eta_{j}\right) h-\pi\right)+f\left(\left(k+\frac{1}{2}-\eta_{j}\right) h-\pi\right)\right] .
$$

Because the basic rule $Q(f)$ in (3.14) is symmetric, we observe that the composite rule $Q_{h}(f)$ is symmetric with respect to the origin $t=0$. More precisely, $Q_{h}(f)$ is of the form (with a certain integer $P$ )

$$
Q_{h}(f)=h \sum_{l=1}^{P} \tilde{w}_{l}\left(f\left(\tau_{l}\right)+f\left(-\tau_{l}\right)\right)
$$

where $0 \leq \tau_{l}<\pi, 1 \leq l \leq P$.

Now, our discretised method is defined as follows: find $u_{h} \in T_{e}^{h}$ such that

$$
\left(L_{e, h} u_{h}, v\right)_{h}=(f, v)_{h}, \quad v \in S_{e}^{d} .
$$

We briefly describe the matrix equation corresponding to (3.18). Having choosen a basis $v_{k, e}, 0 \leq k \leq L$, of $S_{e}^{d}$, we find that (3.18) is equivalent to the $(L+1) \times(L+1)$ system of equations

$$
\sum_{j=L+1}^{2 L+1} a_{k j} u_{h}\left(t_{j}\right)=f_{k}, \quad 0 \leq k \leq L
$$

where

$$
\begin{gathered}
a_{k j}=-\frac{h}{\pi} \sum_{l=1}^{P} \tilde{w}_{l} \log \left|a\left(\tau_{l}\right)-a\left(t_{j}\right)\right| \overline{v_{k, e}\left(\tau_{l}\right)}, \\
f_{k}=\sum_{l=1}^{P} \tilde{w}_{l} f\left(\tau_{l}\right) \overline{v_{k, e}\left(\tau_{l}\right)},
\end{gathered}
$$

and where the asterisk in (3.19) indicates that the last term of the sum is to be halved. In deriving (3.20), (3.21) we have used the fact that all the functions appearing in (3.18) are even. 
REMARK 3.1. It is worth pointing out that (3.18) really is a quadrature method. Namely, consider the quadrature method: find the values $u_{j}, L+1 \leq j \leq$ $2 L+1$ such that

$$
\sum_{j=L+1}^{2 L+1} a_{k j} u_{j}=f_{k}, \quad 0 \leq k \leq L,
$$

where the unknowns $u_{j}$ can be considered as approximations $u_{j} \simeq u\left(t_{j}\right)$. However (3.18) is equivalent to (3.22), since the interpolation problem: for given numbers $u_{j}, L+1 \leq j \leq 2 L+1$, find $u_{h} \in T_{e}^{h}$ such that

$$
u_{h}\left(t_{j}\right)=u_{j}, \quad L+1 \leq j \leq 2 L+1,
$$

is uniquely solvable. The solution $u_{h}$ of (3.23) is given by

$$
u_{h}(t)=\frac{1}{2} a_{0}+\sum_{n=1}^{L} a_{n} \cos n t, \quad-\pi \leq t \leq \pi,
$$

where

$$
a_{n}=\frac{4}{N} \sum_{k=1}^{L+1} u_{L+k} \cos \frac{n \pi}{N}(2 k-1) .
$$

Remark 3.2. Our analysis in Sections 4 and 5 will show (under some additional assumptions on the quadrature rule $Q(f)$ that the problem (3.18) is uniquely solvable if the mesh parameter $h$ is small enough. Thus, for small $h$, we have the unique solvability for the quadrature method (3.22) as well.

REMARK 3.3. The use of the cosine interpolation, described by (3.23)-(3.25), which in effect extends (3.22) to (3.18), is a theoretical tool which, besides the unique solvability, yields also stability results and convergence estimates for the error.

\section{A special case}

Here we consider the special case where the arc $\Gamma$ is the interval $\left[-2 e^{-1}\right.$, $\left.2 e^{-1}\right]$. For this particular arc we have $L_{e}=A_{e}$ and $L_{e, h}=A_{e, h}$, where

$$
\left(A_{e, h} u\right)(t)=-\frac{h}{2 \pi} \sum_{j=0}^{N} \log \left[2 e^{-1}\left|\cos t-\cos t_{j}\right|\right] u\left(t_{j}\right) .
$$

Problem (3.18) now becomes

$$
u_{h} \in T_{e}^{h}:\left(A_{e, h} u_{h}, v\right)_{h}=(f, v)_{h}, \quad v \in S_{e}^{d} .
$$


We are going to show that this problem is equivalent to the problem

$$
u_{h} \in T^{h}:\left(A_{h} u_{h}, v\right)_{h}=(f, v)_{h}, \quad v \in S^{d},
$$

assuming that $f$ is an even function. In (4.3) the operator $A_{h}$ is the trapezoidal approximation of the operator $A$ given in (2.11), that is

$$
\left(A_{h} u\right)(t)=-\frac{h}{\pi} \sum_{j=0}^{N}{ }^{\prime \prime} \log \left|2 e^{-\frac{1}{2}} \sin \left(\frac{t-t_{j}}{2}\right)\right| u\left(t_{j}\right) .
$$

Observe that the function $\left(A_{h} u\right)(t)$ is continuous for $t \neq t_{j}$. Now, let $C_{2 \pi, e}(\mathbb{R})$ and $C_{2 \pi, 0}(\mathbb{R})$, be the space of all continuous $2 \pi$-periodic even and odd functions respectively on $\mathbb{R}$.

We shall need

LEMMA 4.1. The following assertions are true:

(i) $A_{h} u$ is even, if $u \in C_{2 \pi, e}(\mathbb{R})$,

(ii) $A_{h} u$ is odd, if $u \in C_{2 \pi, o}(\mathbb{R})$,

(iii) $A_{h} u=A_{e, h} u$, if $u \in C_{2 \pi, e}(\mathbb{R})$.

Proof. We use the convention

$$
\sum_{j=0}^{L} c_{j}=\frac{1}{2} c_{0}+\sum_{j=1}^{L} c_{j} .
$$

By the symmetry property $t_{N-j}=-t_{j}$ of our mesh, we have for even functions $u$

$$
\left(A_{e, h} u\right)(t)=-\frac{h}{\pi} \sum_{j=0}^{L} \log \left[2 e^{-1}\left|\cos t-\cos t_{j}\right|\right] u\left(t_{j}\right) .
$$

For the function $\left(A_{h} u\right)(t)$, we may split the sum in (4.4) into two terms

$$
\begin{aligned}
\left(A_{h} u\right)(t)= & -\frac{h}{\pi} \sum_{j=0}^{L} \log \left|2 e^{-\frac{1}{2}} \sin \left(\frac{t-t_{j}}{2}\right)\right| u\left(t_{j}\right) \\
& -\frac{h}{\pi} \sum_{j=0}^{L}{ }^{\prime} \log \left|2 e^{-\frac{1}{2}} \sin \left(\frac{t+t_{j}}{2}\right)\right| u\left(-t_{j}\right) .
\end{aligned}
$$

If $u \in C_{2 \pi, e}(\mathbb{R})$, we thus obtain

$$
\left(A_{h} u\right)(t)=-\frac{h}{\pi} \sum_{j=0}^{L} \log \left|4 e^{-1} \sin \left(\frac{t-t_{j}}{2}\right) \sin \left(\frac{t+t_{j}}{2}\right)\right| u\left(t_{j}\right) .
$$


[11] A discrete method for the logarithmic-kernel integral equation on an open arc

Assertion (iii) follows from (4.5), (4.7) by the formula

$$
\sin a \cdot \sin b=\frac{1}{2}[\cos (a-b)-\cos (a+b)] \text {. }
$$

Assertions (i), (ii) can be seen by (4.6).

Now we are able to prove

THEOREM 4.1. Assume that the problem (4.3) is uniquely solvable and that $f \in C_{2 \pi, e}(\mathbb{R})$. Then the problems (4.2) and (4.3) are equivalent.

Proof. By Lemma 4.1(iii), the problem (4.2) can be written as

$$
u_{h} \in T_{e}^{h}:\left(A_{h} u_{h}, v\right)_{h}=(f, v)_{h}, \quad v \in S_{e}^{d} .
$$

Since $N$ is an odd integer, we can write the space $T^{h}$ (analogously with (3.7)) as the direct sum $T^{h}=T_{e}^{h}+T_{o}^{h}$, where $T_{o}^{h}=\left\{u \in T^{h}: u\right.$ is odd $\}$. If $u_{h} \in T^{h}, v \in S^{d}$, we correspondingly write

$$
\begin{aligned}
u_{h} & =u_{h, e}+u_{h, o}, \quad u_{h, e} \in T_{e}^{h}, u_{h, o} \in T_{o}^{h}, \\
v & =v_{e}+v_{o}, \quad v_{e} \in S_{e}^{d}, \quad v_{o} \in S_{o}^{d} .
\end{aligned}
$$

If we have an even function $\tilde{u}$ and an odd function $\tilde{v}$, then the symmetry property of the rule $Q_{h}$ in (3.17) yields

$$
(\tilde{u}, \tilde{v})_{h}=Q_{h}(\tilde{u} \tilde{v})=0
$$

(and similarly $(\tilde{v}, \tilde{u})_{h}=0$ ). By Lemma 4.1 , we thus have

$$
\left(A_{h} u_{h, e}, v_{o}\right)_{h}=\left(A_{h} u_{h, o}, v_{e}\right)_{h}=0
$$

for all $u_{h} \in T^{h}, v \in S^{d}$. Hence we obtain

$$
\left(A_{h} u_{h}, v\right)_{h}=\left(A_{h} u_{h, e}, v_{e}\right)_{h}+\left(A_{h} u_{h, o}, v_{o}\right)_{h}
$$

for all $u_{h} \in T^{h}, v \in S^{d}$.

Now, if $u_{h} \in T_{e}^{h}$ is a solution of (4.8) then $u_{h, e}=u_{h}$ and $u_{h, o}=0$, so by $(4.10)$

$$
\left(A_{h} u_{h}, v\right)_{h}=\left(A_{h} u_{h}, v_{e}\right)_{h}=\left(f, v_{e}\right)_{h}=(f, v)_{h}
$$

for all $v \in S^{d}$. Thus $u_{h}$ is also a solution of the problem (4.3).

Conversely, suppose that $u_{h} \in T^{h}$ satisfies (4.3). Then we have by (4.10)

$$
\left(A_{h} u_{h, e}, v_{e}\right)_{h}+\left(A_{h} u_{h, o}, v_{o}\right)_{h}=(f, v)_{h}=\left(f, v_{e}\right)_{h}
$$

for all $v \in S^{d}$. By choosing $v=v_{o}$, we find

$$
\left(A_{h} u_{h, o}, v_{o}\right)_{h}=0 \text {. }
$$


Now, combining (4.9) and (4.12) we obtain

$$
\left(A_{h} u_{h, o}, v\right)_{h}=0 \quad, \quad v \in S^{d} .
$$

Since the problem (4.3) is assumed to be uniquely solvable, we conclude that $u_{h, o}=0$, and therefore $u_{h}=u_{h, e} \in T_{e}^{h}$. In particular, $u_{h}$ is a solution of (4.2).

Methods of the form (4.3) were studied by Sloan and Burn in [13]. These authors consider the general case of the discrete inner product which is defined by using the composite rule (similar to (3.16)) composed from the basic quadrature rule

$$
Q(f)=\sum_{j=1}^{J} \omega_{j} f\left(\xi_{j}\right)
$$

for the interval $[0,1]$. Here $\sum_{j=1}^{J} \omega_{j}=1, \omega_{j}>0$, and $0<\xi_{1}<\cdots<\xi_{J}<$ 1. The quadrature rule (3.14) becomes a special case of (4.14) if we take $J=2 M$ and define

$$
\left\{\begin{array}{l}
\xi_{j}=\frac{1}{2}-\eta_{M+1-j}, \quad \omega_{j}=w_{M+1-j}, \\
\xi_{M+j}=\frac{1}{2}+\eta_{j}, \quad \omega_{M+j}=w_{j}
\end{array}\right.
$$

for the indices $1 \leq j \leq M$. (Note that $\xi_{M}=\xi_{M+1}$ if $\eta_{1}=0$.)

Now, referring to the terminology of [13], we say that the method (4.2) is stable and of order $p$ if and only if the related method (4.3) has these properties. By the convergence result of [13] for the circle and by the equivalence of the methods (4.2) and (4.3), we obtain

THeOREM 4.2 (Interval). Assume that the arc $\Gamma$ is the interval $\Gamma=\left[-2 e^{-1}\right.$, $2 e^{-1}$ ] and let $u \in H_{e}^{s}$ be the solution of (2.4) (with $L_{e}=A_{e}$ ). Moreover, suppose that the method (4.2) is stable and of order $p$. Then, for all $f \in$ $C_{2 \pi, e}(\mathbb{R})$, there exists a unique solution $u_{h} \in T_{e}^{h}$ of the problem (4.2) and we have the asymptotic error estimate

$$
\left\|u-u_{h}\right\|_{t} \leq c h^{s-t}\|u\|_{s}
$$

if

$$
-1 \leq t \leq s \leq t+p, \quad s>-\frac{1}{2} .
$$

By Sobolev's embedding theorem we obtain for the maximum norm

Corollary 4.1. Assume that $u \in H_{e}^{s}$ with $s \geq \frac{1}{2}+\epsilon$, where $\epsilon>0$. Then we have the pointwise estimate

$$
\left|u-u_{h}\right|_{\infty} \leq c h^{\min \left\{s-\left(\frac{1}{2}+\epsilon\right), p\right\}}\|u\|_{\min \left\{s,\left(\frac{1}{2}+\epsilon\right)+p\right\}} .
$$


In approximating linear functionals of $u$, error estimates with respect to the Sobolev norms of the negative order are particularly useful. Therefore we formulate separately the following result:

COROLlary 4.2. With the assumptions of Theorem 4.2, the estimate

$$
\left\|u-u_{h}\right\|_{-1} \leq c h^{s+1}\|u\|_{s}
$$

holds, provided

$$
-\frac{1}{2}<s \leq p-1 .
$$

EXAMPLE 4.1. As a concrete example of the quadrature methods in (4.2), we can make the following choice:

$$
M=1, \quad \eta_{1}=\frac{1}{3}, \quad w_{1}=\frac{1}{2} .
$$

In terms of the quadrature rule (4.14) this corresponds to the values

$$
J=2, \quad \xi_{1}=\frac{1}{6}, \quad \xi_{2}=\frac{5}{6}, \quad \omega_{1}=\omega_{2}=\frac{1}{2} .
$$

By [13] it is known that the quadrature method (4.3) with (4.22) is stable and of order $p=3$.

In Section 5 we are going to show that the above convergence results are true also for the general open arc. Therefore we shall compare our result to that of Atkinson and Sloan [1, Theorem 6] already at this point. Under the assumption $u \in H_{e}^{s}$ with $s>\frac{1}{2}+\epsilon$, where $\epsilon>0$, Atkinson and Sloan find an approximation $u_{h} \in T_{e}^{h}$ such that

$$
\left|u-u_{h}\right|_{\infty} \leq c h^{s-\left(\frac{1}{2}+\epsilon\right)}\|u\|_{s} .
$$

In particular, the convergence becomes arbitrarily high (in the powers of $h$ ) if the solution $u$ is smooth. By (4.18) we have an analogous result, except that the convergence is limited to the order $O\left(h^{p}\right)$. On the other hand, our method requires less smoothness of the exact solution in order to be convergent (if the error is measured by suitable Sobolev norms). In addition we can use the estimates (4.19), for example, in approximating the corresponding potential outside of the arc. Thus if

$$
\Phi(x)=-\frac{1}{2 \pi} \int_{-\pi}^{\pi} u(\tau) \log |x-a(\tau)| d \tau
$$

is the potential corresponding to the solution $u$, we have for the approximate potential

$$
\Phi_{h}(x)=-\frac{1}{2 \pi} \int_{-\pi}^{\pi} u_{h}(\tau) \log |x-a(\tau)| d \tau
$$


the estimate

$$
\left|\Phi(x)-\Phi_{h}(x)\right| \leq c h^{s+1}\|u\|_{s},
$$

if $-\frac{1}{2}<s \leq p-1$, with $c$ depending on $x$.

\section{The case of the general open arc}

We begin with a lemma relating the integral operator

$$
(K u)(t)=\int_{-\pi}^{\pi} k(t, \tau) u(\tau) d \tau, \quad t \in[-\pi, \pi],
$$

where $k \in C_{2 \pi}(\mathbb{R} \times \mathbb{R})$ (i.e. $k$ is continuous and $2 \pi$-periodic with respect to each variable), to its trapezoidal approximation

$$
\left(K_{h} u\right)(t)=h \sum_{j=0}^{N} k\left(t, t_{j}\right) u\left(t_{j}\right),
$$

for the particular case in which $u$ is a trigonometric polynomial in $T^{h}$.

LEMma 5.1. Suppose $k \in C_{2 \pi}(\mathbb{R} \times \mathbb{R})$ is m-times continuously differentiable with respect to its second variable, and that the corresponding derivative $k_{\tau}^{(m)}(t, \tau)$ is Hölder continuous with respect to $\tau$ with exponent $\lambda, 0<\lambda<1$. Then the estimate

$$
\left|\left(K-K_{h}\right) u_{h}\right|_{\infty} \leq C h^{m+\lambda}\left\|u_{h}\right\|_{0}
$$

holds for all $u_{h} \in T^{h}$.

Proof (cf. [7, Lemma 1] and [6, Sections 2.3.1-2.3.2]). For $f \in C_{2 \pi}$ we may define the trigonometric interpolation operator $L_{N}$, with $N=2 L+1$, by

$$
L_{N} f \in T^{h}, \quad L_{N} f\left(t_{j}\right)=f\left(t_{j}\right), \quad 0 \leq j \leq N .
$$

Then it is easy to show that, for $u_{h} \in T^{h}, K_{h} u_{h}$ can be written as

$$
\left(K_{h} u_{h}\right)(t)=\int_{-\pi}^{\pi} L_{N \tau}[k(t, \tau)] u_{h}(\tau) d \tau
$$

where $L_{N \tau}[k(t, \tau)]$ stands for the operator $L_{N}$ applied to $k(t, \tau)$ with respect to the second variable $\tau$. The result (5.3) follows from the easily verified fact that the trapezoidal rule with spacing $h=2 \pi / N=2 \pi /(2 L+1)$ is exact for all trigonometric polynomials of degree $\leq 2 L$ : since $L_{N \tau}[k(t, \tau)] u_{h}(\tau)$ is a trigonometric polynomial of degree $\leq 2 L$ for each 
fixed value of $t$, the integral on the right of (5.3) is equal to its trapezoidal approximation

$$
h \sum_{j=0}^{N} L_{N \tau}\left[k\left(t, t_{j}\right)\right] u_{h}\left(t_{j}\right)=h \sum_{j=0}^{N} k\left(t, t_{j}\right) u_{h}\left(t_{j}\right),
$$

and so is equal to $K_{h} u_{h}(t)$.

From (5.1) and (5.3) we now have

$$
\left(K-K_{h}\right) u_{h}(t)=\int_{-\pi}^{\pi}\left(k(t, \tau)-L_{N \tau}[k(t, \tau)]\right) u_{h}(\tau) d \tau .
$$

The Cauchy-Schwarz inequality then gives

$$
\begin{aligned}
\left|\left(K-K_{h}\right) u_{h}\right|_{\infty} & \leq \max _{t} \int_{-\pi}^{\pi}\left|k(t, \tau)-L_{N \tau}[k(t, \tau)] \| u_{h}(\tau)\right| d \tau \\
& \leq \varepsilon_{h}\left\|u_{h}\right\|_{0},
\end{aligned}
$$

where

$$
\begin{aligned}
\varepsilon_{h} & :=\max _{t}\left\|k(t, \tau)-L_{N \tau}[k(t, \tau)]\right\|_{0} \\
& \leq\left(1+\left\|L_{N}\right\|_{C \rightarrow L^{2}}\right) \max _{t} E_{h}(C ; k(t, \cdot)) .
\end{aligned}
$$

Here $\left\|L_{N}\right\|_{C \rightarrow L^{2}}$ stands for the norm of the operator $L_{N}: C \rightarrow L^{2}$, and $E_{h}(C ; k(t, \cdot))$ is the error of best approximation of $k(t, \tau)$ (with fixed $t$ ) by trigonometric polynomials from $T^{h}$ with respect to the uniform norm. Now by Jackson's theorem, $E_{h}(C ; k(t, \cdot))=O\left(h^{m+\lambda}\right)$. Since, as is well known, $\sup _{N}\left\|L_{N}\right\|_{C \rightarrow L^{2}}<\infty$, we have $\varepsilon_{h}=O\left(h^{m+\lambda}\right)$, and the proof is complete.

If $k(t, \tau)$ is also differentiable with respect to its first variable $t$, then similar results may be obtained for the derivatives of $\left(K-K_{h}\right) u_{h}$. By proceeding in this way we may obtain the following result, which was already found by Saranen and Sloan in [10].

LEMMA 5.2. Let $k \in C_{2 \pi, e}^{\infty}(\mathbb{R} \times \mathbb{R})$. Then, for any $s, t \in \mathbb{R}$ and $\delta>0$, there exists a constant $C>0$ (independent of $u_{h} \in T_{e}^{h}$ ) such that

$$
\left\|\left(K-K_{h}\right) u_{h}\right\|_{t} \leq C h^{\delta}\left\|u_{h}\right\|_{s}
$$

holds for all $u_{h} \in T_{e}^{h}$.

The approximation property (5.4) provides the essential tool to discuss the case of the general open arc. 
TheOREM 5.1. Assume that $\Gamma$ is a smooth open arc such that its capacity differs from 1. Let $u \in H_{e}^{s}$ be the solution of (2.4) and suppose the method (4.2) is stable and of order $p$. Then, for all $f \in C_{2 \pi, e}(\mathbb{R})$ and all $h$ sufficiently small, there exists a unique solution $u_{h} \in T_{e}^{h}$ of the problem (3.18) and we have the asymptotic error estimate

$$
\left\|u-u_{h}\right\|_{t} \leq c h^{s-t}\|u\|_{s}
$$

if $-1 \leq t \leq s \leq t+p, s>-\frac{1}{2}$.

Proof (cf. [10]). Assume initially that (3.18) has a solution $u_{h}$. By writing $f=L_{e} u=\left(A_{e}+B_{e}\right) u$ and

$$
L_{e, h}=A_{e, h}+B_{e, h},
$$

(3.18) becomes

$$
\left(A_{e, h} u_{h}, v\right)_{h}=\left(A_{e}\left(u+A_{e}^{-1}\left(B_{e} u-B_{e, h} u_{h}\right)\right), v\right)_{h}
$$

for all $v \in S_{e}^{d}$. By Theorem 4.2 we thus have

$$
\left\|u+A_{e}^{-1}\left(B_{e} u-B_{e, h} u_{h}\right)-u_{h}\right\|_{t} \leq c h^{s-t}\left\|u+A_{e}^{-1}\left(B_{e} u-B_{e, h} u_{h}\right)\right\|_{s} .
$$

Now, the operator $I+A_{e}^{-1} B_{e}: H_{e}^{t} \rightarrow H_{e}^{t}$ is an isomorphism, so we obtain

$$
\begin{aligned}
\left\|u-u_{h}\right\|_{t} \leq & c\left\|\left(I+A_{e}^{-1} B_{e}\right)\left(u-u_{h}\right)\right\|_{t} \\
\leq & c\left\|u-u_{h}+A_{e}^{-1}\left(B_{e} u-B_{e, h} u_{h}\right)\right\|_{t} \\
& +c\left\|A_{e}^{-1}\left(B_{e}-B_{e, h}\right) u_{h}\right\|_{t} .
\end{aligned}
$$

Combining (5.6) and (5.7), we have

$$
\begin{aligned}
\left\|u-u_{h}\right\|_{t} \leq & c h^{s-t}\left\|u+A_{e}^{-1}\left(B_{e} u-B_{e, h} u_{h}\right)\right\|_{s}+c\left\|A_{e}^{-1}\left(B_{e}-B_{e, h}\right) u_{h}\right\|_{t} \\
\leq & c h^{s-t}\left(\|u\|_{s}+\left\|A_{e}^{-1} B_{e}\left(u-u_{h}\right)\right\|_{s}+\left\|A_{e}^{-1}\left(B_{e}-B_{e, h}\right) u_{h}\right\|_{s}\right) \\
& +c\left\|A_{e}^{-1}\left(B_{e}-B_{e, h}\right) u_{h}\right\|_{t} .
\end{aligned}
$$

Since the operator $B_{e}$ is infinitely smoothing, we conclude that

$$
\left\|A_{e}^{-1} B_{e}\left(u-u_{h}\right)\right\|_{s} \leq c\left\|u-u_{h}\right\|_{-1} .
$$

Applying Lemma 5.2 to the operator $B_{e}$ with the kernel $b_{e} \in C_{2 \pi, e}^{\infty}(\mathbb{R} \times \mathbb{R})$, we find for all $\delta>0$

$$
\begin{gathered}
h^{s-t}\left\|A_{e}^{-1}\left(B_{e}-B_{e, h}\right) u_{h}\right\|_{s}+\left\|A_{e}^{-1}\left(B_{e}-B_{e, h}\right) u_{h}\right\|_{t} \\
\leq c h^{\delta}\left\|u_{h}\right\|_{-1} \leq c h^{\delta}\left(\|u\|_{-1}+\left\|u-u_{h}\right\|_{-1}\right) .
\end{gathered}
$$


Choosing $\delta \geq s-t$ and combining (5.8)-(5.10), we obtain

$$
\left\|u-u_{h}\right\|_{t} \leq c h^{s-t}\left(\|u\|_{s}+\left\|u-u_{h}\right\|_{-1}\right)
$$

for all $-1 \leq t \leq s \leq t+p, s>-\frac{1}{2}$.

Taking $t=-1$, we have by (5.11), for small $h$

$$
\left\|u-u_{h}\right\|_{-1} \leq c h^{s+1}\|u\|_{s} \text {. }
$$

Inserting (5.12) back into (5.11), we have the inequality (5.5). Above we have assumed that the solution $u_{h} \in T_{e}^{h}$ exists. But (5.5) with $u$ replaced by zero establishes the uniqueness of the solution for small $h$, and from this the existence follows immediately.

\section{Acknowledgements}

The support of the Australian Research Council is gratefully acknowledged. One of us (IHS) was also partly supported by the US Army Research Office through the Mathematical Sciences Institute of Cornell University.

\section{References}

[1] K. E. Atkinson and I. H. Sloan, "The numerical solution of first-kind integral equations on smooth open arcs", Math. Comp. 56 (1991) 119-139.

[2] M. Costabel, V. J. Ervin and E. P. Stephan, "On the convergence of collocation methods for Symm's integral equations on open curves", Math. Comp. 51 (1988) 167-179.

[3] S. Joe and $Y$. Yan, “A collocation method using cosine mesh grading for Symm's equation on the interval $(-1,1)$ ", Preprint, April 1990, University of the New South Wales, Sydney.

[4] H. Multhopp, "Die Berechnung der Auftriebsverteilung von Tragflügeln", LuftfahrtForschung XV (4) (1938) 153-169.

[5] S. Prössdorf and A. Rathsfeld, "Quadrature methods for strongly elliptic Cauchy singular integral equations on an interval", Operator Theory: Advanced and Applications, 41 (Birkhäuser Verlag, Basel, 1989) 435-471.

[6] S. Prössdorf and B. Silbermann, Projektionsverfahren und die näherungsweise Lösung singulärer Gleichungen, Teubner-Texte für Mathematik (Teubner Verlagsges., Leipzig, 1977).

[7] S. Prössdorf and I. H. Sloan, "Quadrature method for singular integral equations on closed curves", Numer. Math. 61 (1992) 543-559.

[8] A. Rathsfeld, "Quadraturformelverfahren für Mellin-Operatoren nullter Ordnung", Math. Nachr. 137 (1988) 321-354.

[9] J. Saranen, "The modified quadrature method for logarithmic-kernel integral equations on closed curves", J. Integral Equations Appl. 3(4) (1991) 575-600.

[10] J. Saranen and I. H. Sloan, "Quadrature methods for logarithmic-kernel integral equations on closed curves", IMA J. Numer. Anal. 12 (1992) 167-187.

[11] M. Schleiff, "Untersuchung einer linearen singulären Integrodifferentialgleichung der Tragflügeltheorie", Wiss. Z. der Univ. Halle XVII 68 M, (6) (1968) 981-1000. 
[12] M. Schleiff, “Über Näherungsverfahren zur Lösung einer singulären linearen Integrodifferentialgleichung", ZAMM 48 (1968) 477-483.

[13] I. H. Sloan and B. J. Burn, "An unconventional quadrature method for logarithmic-kernel integral equations on closed curves", J. Integral Equations Appl. 4(1) (1992) 117-151.

[14] I. H. Sloan and A. Spence, "The Galerkin method for integral equations of the first kind with logarithmic kernel: Theory", IMA J. Numer. Anal. 8 (1988) 105-122.

[15] I.H. Sloan and E. P. Stephan, "Collocation with Chebyshev polynomials for Symm's integral equation on an interval", J. Austral. Math. Soc. Ser. B (to appear).

[16] J. Weissinger, "Über Integrodifferentialgleichungen vom Typ der Prandtlschen Tragflügelgleichung", Math. Nachr. 3 (1950) 316-326.

[17] Y. Yan and I. H. Sloan, "On integral equations of the first kind with logarithmic kernel", J. Integral Equations Appl. 1 (1988) 549-579. 